\title{
The MULTI spectrometer for measurement of $\beta$ - decay process in exotic nuclei
}

\author{
Ivan Siváček ${ }^{1,2}$, Sergey Stukalov ${ }^{1}$, Yuri Sobolev ${ }^{1}$, Dmitry Testov $^{1}$, Vladimir Smirnov ${ }^{1}$, Yuri \\ Penionzhkevich ${ }^{1,3}$ and Zhasulan Zeinulla ${ }^{1,4}$ \\ ${ }^{1}$ Flerov Laboratory of Nuclear Reactions, Joint Institute for Nuclear Research, 141980 Dubna, Russia \\ ${ }^{2}$ Nuclear Physics Institute of the Czech Academy of Sciences, 25068 Řež, Czech Republic \\ ${ }^{3}$ National Research Nuclear University, 115409 Moscow, Russia \\ ${ }^{4}$ Institute of Nuclear Physics, Almaty, Kazakhstan \\ sivacek@ujf.cas.cz
}

\begin{abstract}
The construction of the portable spectrometer, MULTI, is designed for the measurement of $\beta$ - $\gamma$-neutron coincidences on beams of radioactive exotic nuclei. It will be used in measurements of the $\beta$-decay process of neutronrich nuclei with large $Q_{\beta}$ values accessible in radioactive beams facilities. The experimental technique includes the measurement of the ratios between gamma-ray emission and multi-neutron emission, following beta-decay of radioactive nuclei implanted into an active target. The construction of a gamma-ray spectrometer based on highvolume $\mathrm{CeBr}_{3}+\mathrm{NaI}(\mathrm{Tl})$ phoswich scintillation detectors is introduced. Results of the measurements of its fundamental characteristics - total detection efficiency, peak efficiency and Compton suppression effect with a ${ }^{60} \mathrm{Co}$ source are presented. Design of the neutron counter array, consisting of 40 proportional counters filled with ${ }^{3} \mathrm{He}$ gas, was optimized with Monte Carlo simulations. Results were validated with a ${ }^{252} \mathrm{Cf}$ spectroscopic source, leading to expected detection efficiency of $\sim 35 \%$ for neutrons with energy $<1 \mathrm{MeV}$. Further development plans are discussed.
\end{abstract}

Keywords - Monte Carlo simulations, Phoswich detector, gamma-ray spectrometer, ${ }^{3} \mathrm{He}$ neutron counters.

\section{INTRODUCTION}

$\mathrm{T}^{\mathrm{s}}$ HE MULTI spectrometer was developed as a setup for measurement of the total reaction cross section with radioactive beams [1]. Measurements were carried out on the ACCULINNA fragment separator beam in the Flerov Laboratory of Nuclear Reactions, Joint Institute for Nuclear Research in Dubna (FLNR JINR) [2]. The Nuclear Physics Institute of the Czech Academy of Sciences (NPI CAS) is involved in the collaboration since 2012 [3]. Fundamental properties of the spectrometer, important for the evaluation of results, include detection efficiency of the gamma-ray spectrometer and registration efficiency for nuclear reaction events as a function of gamma-ray energy and multiplicity were earlier evaluated by Monte Carlo simulations in Geant3 [1] and later Geant4 [4]. The spectrometer consisted of an in-beam telescope for measurement of beam characteristics and a gamma-ray spectrometer for identification of nuclear reaction events by prompt gamma-rays. A lot of effort was put into development and optimization of the active collimators [5] and the method of measurement with an active target. Energy dependence of the total reaction cross section for light neutronrich nuclei ${ }^{6,8} \mathrm{He},{ }^{9,11} \mathrm{Li}$ was measured [6,7], results were successfully interpreted theoretically by the time-dependent Schrödinger equation for ${ }^{11} \mathrm{Li}+{ }^{28} \mathrm{Si}$ [8]. The measurement technique includes detection of prompt gamma-rays accompanying the nuclear reactions, which provide a tag for a reaction event. The MULTI spectrometer was upgraded to enhance registration efficiency for nuclear reaction events and lower its dependency on multiplicity of prompt gamma-rays [9]. Monte Carlo results were verified using measurements with a ${ }^{60} \mathrm{Co}$ spectroscopic source for gamma-ray multiplicity up to $\mathrm{M}_{\gamma} \leq 5$.

Possibilities of upgrading the MULTI spectrometer with novel high-resolution detectors were analyzed by Monte Carlo method, aimed to improve detection and registration efficiency with possible gamma-ray spectroscopy measurements for identification of the reaction channels [10]. The spectrometer was upgraded with $\mathrm{CeBr}_{3}+\mathrm{NaI}(\mathrm{Tl})$ phoswich scintillation detectors and the electronics migrated from CAMAC to VME digital acquisition standard. Measurements with an active ${ }^{28} \mathrm{Si}$ target were already carried out in 2019, and unfortunately, they can be completed only after the upgrade of the U400M cyclotron in FLNR JINR in 2022.

The recently commissioned fragment separator ACCULINNA-2 offers an opportunity to make measurements on secondary beams of higher purity with lower background [11]. It offers isotopes of light, neutron-rich nuclei, including isotopes with weakly bound neutrons in the neutron halo and neutron skin. Pygmy dipole resonance (PDR) is defined as collective oscillations of the neutron halo against the inert core of the nucleus and is directly related to these phenomena, which can also be studied with gamma-ray spectroscopy methods. Reconfiguration of the detectors for gamma-ray spectroscopy measurements was the subject of Monte Carlo simulations. These exotic nuclei are $\beta^{-}$emitters, often with large $Q_{\beta}$ values, emitting multiple neutrons and gamma-rays following $\beta$-decay. Based on our collaboration with Institut Physique Nucleaire (IPN) in Orsay, France on neutron counter TETRA [12], the neutron counter assembly with proportional counters filled with ${ }^{3} \mathrm{He}$ gas was introduced into simulations. We are preparing the 
MULTI spectrometer for $\beta$-gated gamma-ray spectroscopy and multi-neutron emission measurements, similar to our measurements on ${ }^{82,83,84} \mathrm{Ga}$ isotopes in collaboration with IPN Orsay [13]. We will follow in search of properties of weakly bound neutrons in the region of light neutron-rich nuclei, accessible on the ACCULINNA-2 fragment separator. For this purpose, the correctness of Monte Carlo simulations must be verified with measurements. Initial measurements of the discussed assembly were carried out to validate Monte Carlo simulations of gamma-ray spectrometer and neutron counter array. Results of the measurements are compared to the simulations and discussed.

\section{THE MULTI SPECTROMETER}

Two methods are widely used for the PDR studies: the investigated nuclei can be excited with an external source of radiation, or can be studied in the decay process leading to the excited states of daughter nucleus. We propose the second mentioned method. The process of de-excitation of an implanted exotic nucleus after $\beta$-decay can go through multineutron emission, or trough emission of gamma rays. As proposed for ${ }^{134} \mathrm{Sn}[14]$, energy of the $\beta$-particle determines the mode of de-excitation. Similar studies including measurement of $\beta$-decay strength function are being prepared. The new spectrometer, MULTI, is now developed for measurement of the $\beta$-decay process in neutron-rich nuclei with large $Q_{\beta}$ values. It will be able to measure coincidences between emission of $\beta$ particles, gamma-rays, and multiple neutrons from the decay of the selected radioactive nucleus implanted into the active target.

The MULTI spectrometer consists of an in-beam multidetector telescope with active target, $4 \pi \beta$-detector, a highefficiency gamma-ray spectrometer, and a $4 \pi$ assembly of ${ }^{3} \mathrm{He}$ neutron counters. Current geometry of the setup was optimized with the Monte Carlo method using the software toolkit Geant4 [15-17]. Scheme of the spectrometer, consisting of a gammaray detector array and a neutron counter assembly, is shown in figure 1. Photo of the neutron counters assembly is shown on figure 2. The detector for $\beta$-particles, which will be located in the center of the setup around the active target, is currently being developed.

The in-beam multi-detector telescope is designed for beam transmission measurement and identification of secondary beam particles. It consists of two active collimators, a $\mathrm{dE}_{0}$ silicon detector, and an active target. Active collimators with plastic scintillator define the irradiated area of the target and provide information about beam transmission and time of flight (TOF) for secondary beam projectiles. Particles are identified by a dE-TOF method in a $243 \mu \mathrm{m}$ thick silicon $\mathrm{dE}_{0}$ detector. A detailed description is given in [9]. The active target is designed for the implantation of secondary beam nuclei and for measurements of $\beta$-particles from decay with pulse-shape analyzing electronics. The energy of the secondary beam below $10 \mathrm{MeV} / \mathrm{u}$ will be adjusted with polyethylene absorbers.

The active target will be surrounded by a $\beta$-particle detector covering a large solid angle, made of optically connected fast (plastic) and slow (crystalline) scintillator phoswich. It will be designed to distinguish between high and low energy $\beta$ - particles, using pulse-shape discrimination techniques.

Validation of the Geant 4 results for neutrons and gammarays is necessary for finalization of the detector design. This work is dedicated to the measurement of fundamental properties of gamma-ray spectrometer and validation of the Geant 4 model of the setup for gamma-rays and neutrons. It will help with optimization of the spectrometer geometry for measurements on secondary beams of different facilities as well as with development of the $4 \pi \beta$-detector.

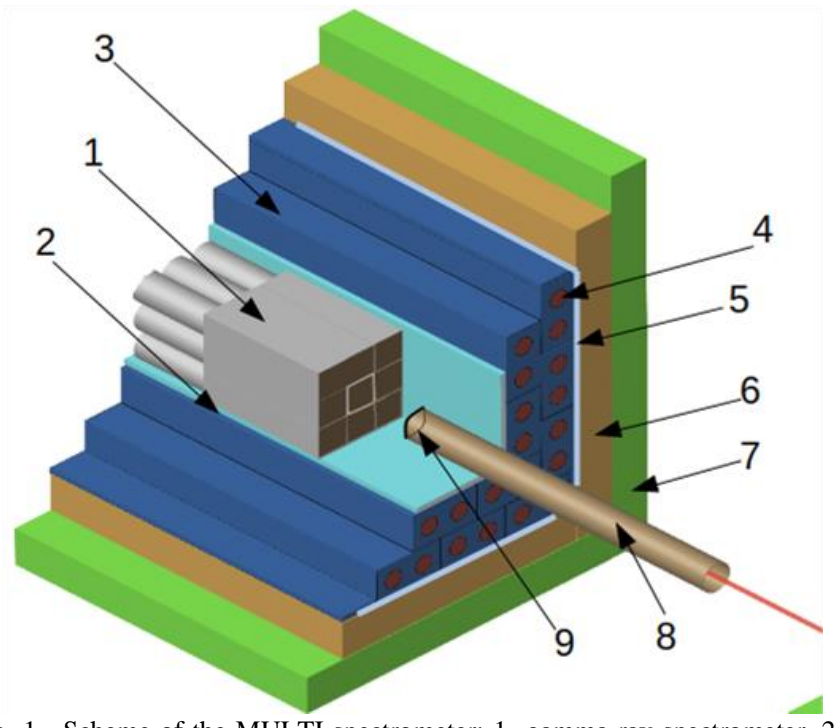

Fig. 1. Scheme of the MULTI spectrometer: 1- gamma-ray spectrometer, 2spectrometer frame, 3- polyethylene blocks, 4- 3He neutron counters, 5- Al frame, 6 - high-density polyethylene, 7 - borated polyethylene $8 \%, 8$ - beam pipe, 9- place for active target and $\beta$-detector.

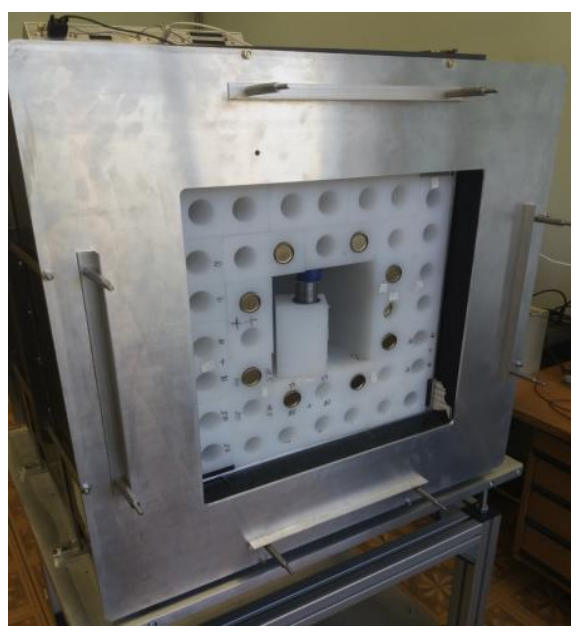

Fig. 2. Photo of neutron counters assembly with central channel for gammaray spectrometer. Nine neutron counters are placed in the inner layer of the polyethylene blocks for detection efficiency measurement.

\section{GAMMA-RAY SPECTROMETER}

The gamma-rays carrying information about nuclear resonances from excited nuclei following beta decay will be registered by high-volume $\mathrm{CeBr}_{3}+\mathrm{NaI}(\mathrm{Tl})$ phoswich scintillation detectors. The detectors are of rectangular shape with a $528 \mathrm{~cm}^{3}$ active volume (fig. 3). The energy resolution of this detector is $3,9 \%(661 \mathrm{keV})$. Its rectangular shape allows for the construction of clusters of detectors of the same type with enhanced characteristics. The PARIS collaboration 
(Photon Array for studies with Radioactive Ion and Stable beams) [18] is connecting different laboratories to build large clusters of phoswich detectors with $\mathrm{CeBr}_{3}$ or $\mathrm{LaBr}_{3}: \mathrm{Ce}$ crystals. We use a $3 \times 3$ cluster of the detectors for our measurements.

The signal from the detector is divided into a fast component $\left(\mathrm{CeBr}_{3}\right.$ crystal) and slow component $(\mathrm{NaI}(\mathrm{Tl})$ crystal) with pulse-shape analyzing electronics. Slow signal can be used for Compton suppression of the gamma-ray spectrum recorded by a high-resolution $\mathrm{CeBr}_{3}$ crystal. Moreover, neighboring detectors in the cluster can serve as Compton suppression detectors as well. It is important in the search for Pygmy resonance states within the background induced by high-energy gamma rays.

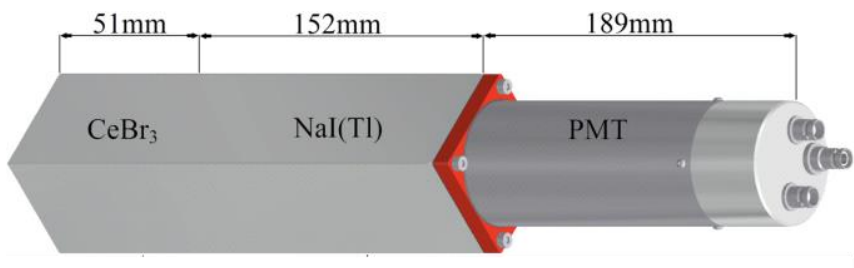

Fig. 3. $\mathrm{CeBr}_{3}+\mathrm{NaI}(\mathrm{Tl})$ phoswich scintillation detector. Dimensions of the crystals are $51 \times 51 \times 51 \mathrm{~mm}\left(\mathrm{CeBr}_{3}\right)$ and $51 \times 51 \times 152 \mathrm{~mm}(\mathrm{NaI}(\mathrm{Tl}))$.

Fundamental characteristics of the spectrometer (peak efficiency, overall registration efficiency over the threshold, and Compton suppression effect) were measured with a ${ }^{60} \mathrm{Co}$ spectroscopic source. The method of measurement is based on tagged gamma-rays [9]. The ${ }^{60} \mathrm{Co}$ source emits two gamma rays in coincidence $-1173,2 \mathrm{keV}$ and $1332,5 \mathrm{keV}$. An external detector was employed to register the $1332,5 \mathrm{keV}$ gamma-ray peak. It initiates the start of the acquisition, while the other $1173,2 \mathrm{keV}$ gamma-ray was registered by the spectrometer. The external detector was a single crystal $\mathrm{CeBr}_{3}$ of the same dimensions placed at $10 \mathrm{~cm}$ distance from the source. Multiple measurements were carried out with the distance of gamma-ray spectrometer at $10 \mathrm{~cm}$ and $40 \mathrm{~cm}$ from source.

The differentiation of the fast and slow components of the pulse is achieved by setting two gates for one pulse by the VME Modular Digital Pulse Processor. The first gate for acquisition of the fast signal had $25 \mathrm{~ns}$, followed by a $600 \mathrm{~ns}$ gate for the slow component of detector impulse. The two-dimensional fastslow signal spectrum of tagged $1173,2 \mathrm{keV}$ gamma-rays is shown on fig. 4. This allows for the use of a $\mathrm{NaI}(\mathrm{Tl})$ crystal of phoswich detector for Compton rejection. The Compton suppression effect is defined as a ratio of mean height of the Compton part of the spectrum with and without Compton rejection. Measurement with tagged gamma-rays at a $40 \mathrm{~cm}$ distance (quasi-parallel rays) showed a 58\% suppression effect for the central detector of the $3 \times 3$ cluster. Geant 4 simulations showed a $66 \%$ effect of Compton suppression under the same conditions. Measurements at $10 \mathrm{~cm}$ distance (fig. 5) showed $63 \%$ suppression effect, while simulations resulted into $72 \%$. Results of the simulations and measurements are compared in Table I.
TABLE I

FUNDAMENTAL CHARACTERISTICS OF THE GAMMA-RAY SPECTROMETER. COMPARISON OF SIMULATION AND MEASUREMENT AT 10 CM AND 40 CM DISTANCE.

\begin{tabular}{lcc}
\hline \hline & $\begin{array}{c}{ }^{60} \mathrm{Co} \\
\text { measurement }\end{array}$ & $\begin{array}{c}\text { Geant } 4 \\
\text { calculation }\end{array}$ \\
\hline Source distance $10 \mathrm{~cm}:$ & & \\
Total efficiency $>400 \mathrm{keV}$ & $8,6 \%$ & $7,7 \%$ \\
Peak efficiency $1173,2 \mathrm{keV}$ & $2,5 \%$ & $3,2 \%$ \\
Compton suppression & $58 \%$ & $66 \%$ \\
Source distance $40 \mathrm{~cm}:$ & & \\
Total efficiency $>400 \mathrm{keV}$ & $0,6 \%$ & $1,2 \%$ \\
Peak efficiency $1173,2 \mathrm{keV}$ & $0,3 \%$ & $0,4 \%$ \\
Compton suppression & $63 \%$ & $72 \%$ \\
\hline \hline
\end{tabular}

The difference between simulations and measurements is mainly due to uncertainty of the source position $(\mathrm{x}, \mathrm{y})$, which was set very roughly. In the simulations, the source is point and has defined coordinates. The distance along the $\mathrm{z}$-axis was measured with uncertainty $\pm 2 \mathrm{~cm}$. Simulations of Compton suppression are more complex task. In the first approximation, rejection can be simulated as an energy deposition in the $\mathrm{NaI}(\mathrm{Tl})$ crystal with some threshold. However, in the measurements, there is always a small part of the slow signal in the fast gate and vice versa. To correctly reproduce measured values with simulations, these "mixture coefficients" should be determined to fit the slope of the measured 2-dimensional spectrum (fig. 4). Previous results with tagged gamma rays achieved excellent agreement with the simulations [9].

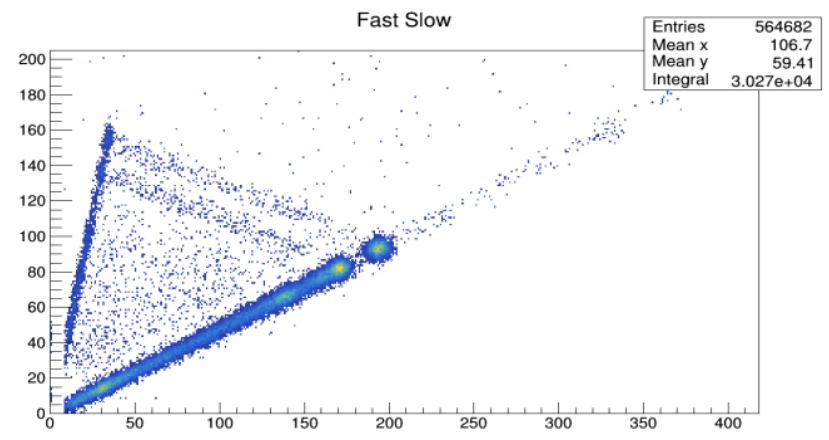

Fig. 4. Two-dimensional spectrum of fast (x-axis) and slow (y-axis) component of detector impulse in arbitrary units.

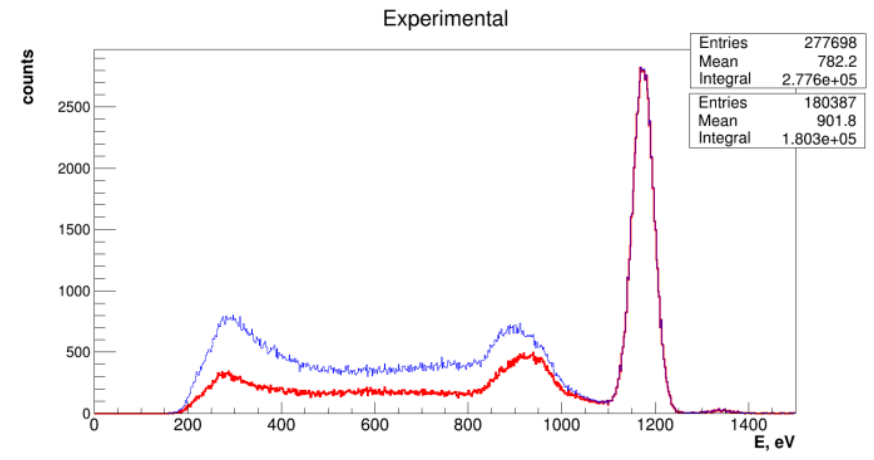

Fig. 5. Compton suppression effect of $\mathrm{NaI}(\mathrm{Tl})$ of the own detector and surrounding detectors for central detector of $3 \times 3$ detector cluster. 


\section{NEUTRON COUNTERS ASSEMBLy}

Neutrons are measured with proportional counters filled with ${ }^{3} \mathrm{He}$ gas placed in high-density polyethylene blocks. They were chosen due to their large volume, high absorption cross-section for thermal neutrons, and zero cross-talk, important for multiplicity measurements. Their modularity allows one to easily adjust geometry, and they show low sensitivity to gamma rays. Neutrons are registered indirectly through the reaction $\mathrm{n}+{ }^{3} \mathrm{He} \rightarrow \mathrm{p}+{ }^{3} \mathrm{H}+764 \mathrm{keV}$.

Materials and design of the setup optimized were with Monte Carlo method with the aim of maximizing the detection efficiency. It is built from polyethylene blocks with dimensions 130x70x600 mm. Each block has two circular apertures, Ø 33 $\mathrm{mm}$, localized symmetrically with center in the middle of block height, at $1 / 4$ and $3 / 4$ of the block width. A total of 20 blocks for 40 neutron counters are situated around the gamma-ray spectrometer in two layers, with 16 and 24 neutron counters respectively (fig. 2). The polyethylene blocks are fixed in a $5 \mathrm{~mm}$ thick aluminum case. The case is surrounded with $50 \mathrm{~mm}$ thick polyethylene moderator layer and a $75 \mathrm{~mm}$ thick $8 \%$ borated polyethylene layer for neutron background suppression. The back panel contains $33 \mathrm{~mm}$ wide circular apertures through all of the layers for inserting neutron counters and cables.

Neutron counters are in the form of $600 \mathrm{~mm}$ long tubes with a $32 \mathrm{~mm}$ diameter. The effective length of the detector is $528 \mathrm{~mm}$, and the pressure of the ${ }^{3} \mathrm{He}$ gas in the mixture is $7 \mathrm{~atm}$. Pre-amplifiers were removed; detectors are directly connected to the neutron-counter electronics module. The module was developed in FLNR, and is similar to the one used in the ALTO facility for the TETRA neutron counter [12]. It uses one highvoltage supply for feeding all counters and a common threshold for pulse-height discrimination. It counts every registered neutron with a $2 \mu \mathrm{s}$ cycle and provides multiplicity measurements in a $128 \mu$ s time frame. It uses TTL logic to communicate with a computer and has an analogue output for every single channel for single spectra acquisition.

Simulation results were verified with measurements with a ${ }^{252} \mathrm{Cf}$ spectroscopic neutron source. The main parameter used for the verification was the detection efficiency of the neutrons in the setup. The aim of the measurements was to validate the results of the simulations, used for optimization of the setup geometry and materials. For this purpose, measurements were performed without a gamma-ray spectrometer inside the setup. A total of nine neutron counters were used for the measurements. The subject of the comparison was detection efficiency in different neutron-counter configurations and measurements with the neutron source placed in center of the setup and at the edges of the polyethylene blocks containing the neutron counters.

Two methods of efficiency evaluation were used. One result is obtained as a ratio of net number of neutrons registered in the setup and the total of the number of emitted neutrons from the ${ }^{252} \mathrm{Cf}$ activity, evaluated for the day of the measurement (fig. 6 yellow triangles). Another method to evaluate detection efficiency is based on the well-known neutron multiplicity distribution $\left(\mathrm{N}_{1}, \mathrm{~N}_{2}, \mathrm{~N}_{3}, \ldots\right)$ of ${ }^{252} \mathrm{Cf}$. The neutron multiplicity ratios $\left(\mathrm{N}_{1} / \mathrm{N}_{2}, \mathrm{~N}_{1} / \mathrm{N}_{3}, \mathrm{~N}_{2} / \mathrm{N}_{3}, \ldots\right)$ depend on the detection efficiency of the setup. With known multiplicity ratios for the source, efficiency of the setup can be extracted from measured ratios (fig. 6 red diamonds). The method is described in [19]. This method is generally more precise, because it does not depend on the activity of the source. However, it is more suitable for large arrays with high registration efficiency.

The threshold on the discriminator, common for all counters, was set to relatively high level of $1,6 \mathrm{~V}$ (maximum amplitude was $\sim 5 \mathrm{~V}$ ) due to electronic noise on the stand. Analysis of spectra from neutron counters showed that part of the spectrum related to neutrons lost is due to threshold in some of the detectors. It is still suitable for the test measurements and verification of the Geant 4 model of the setup; however, improvements must be made before on-beam measurements.

Efficiency was evaluated from the single spectra analysis with variable thresholds, taking into account the full neutron-related spectrum from the proportional counter (fig. 6 blue triangle). The acquired single spectra also allowed us to introduce the equivalent of a $1,6 \mathrm{~V}$ common threshold into the Monte Carlo calculations for each detector on the corresponding position separately (fig. 6 violet triangle). It resulted in the simulated detection efficiency being artificially lowered, taking into account the electronics threshold. Results of the measurements and simulations are shown on fig. 6.

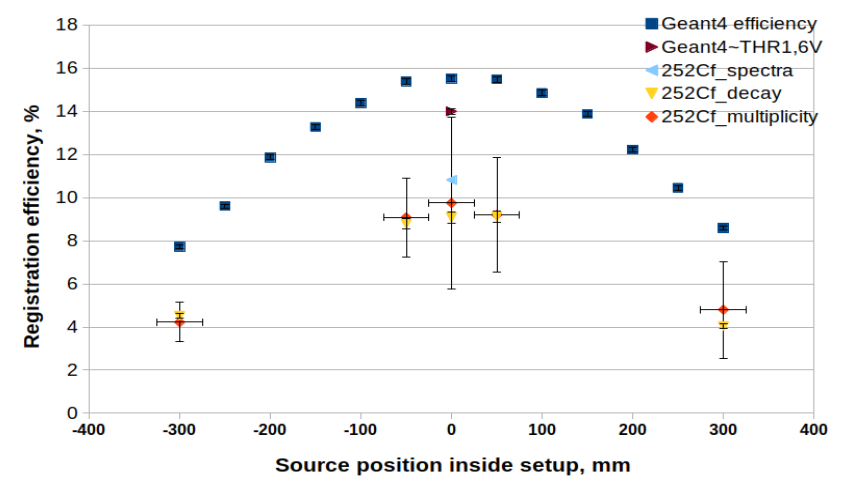

Fig. 6. Comparison of neutron detection efficiency obtained by measurement with ${ }^{252} \mathrm{Cf}$ and Geant 4 calculation. Nine neutron counters were placed in the inner layer moderator blocks (fig. 2).

Detection efficiency results obtained by multiplicity and integral measurements are in good agreement. A source of large uncertainties in the multiplicity measurements are from the variance of the evaluated efficiencies from different neutron multiplicity ratios. This is partially due to the effect of a high common threshold and a low number of neutron counters used for detection of multiple neutrons. Results obtained with single spectra analysis shows that only a relatively small portion of detection efficiency is lost due to the high common threshold.

Comparison of measured values and calculations shows a relatively large discrepancy. Ratios between the detection efficiency measured in the central region of the setup and borders are $\sim 0,5$ for measurements and simulations both. A slightly lower efficiency is observed in the position $-300 \mathrm{~mm}$. It is due to apertures in the back panel of the setup. Detection efficiency obtained with common threshold measurements (integral and multiplicity) compared to artificially lowered Geant4 result gives measurement-to-simulation ratio of 0,67 . 
The same ratio is obtained in comparison of single spectra analysis and Geant 4 simulation results. As a conclusion, Monte Carlo simulations are succeeding in reproducing measurements with a large systematic error of $\sim 33 \%$. Variations of simulation parameters showed that the most important parameter for sensitivity to neutrons is the amount of ${ }^{3} \mathrm{He}$ in the counters, represented by the gas density. Further investigation is required to understand this systematic error and successfully quantitatively reproduce measured values.

In the result a correction factor of 0,67 was introduced to Monte Carlo calculations. Expected energy dependence of the detection efficiency for single neutron in the full assembly of 40 neutron counters is shown on fig. 7. The detection efficiency is $\sim 35 \%$ and it is relatively flat up to $1 \mathrm{MeV}$. Further investigation of the spectrometer characteristics will be carried out with the aim of evaluating the detection efficiency for neutrons in the gamma-ray spectrometer and gamma-rays in the neutron counters with pulse-shape analyzing electronics.

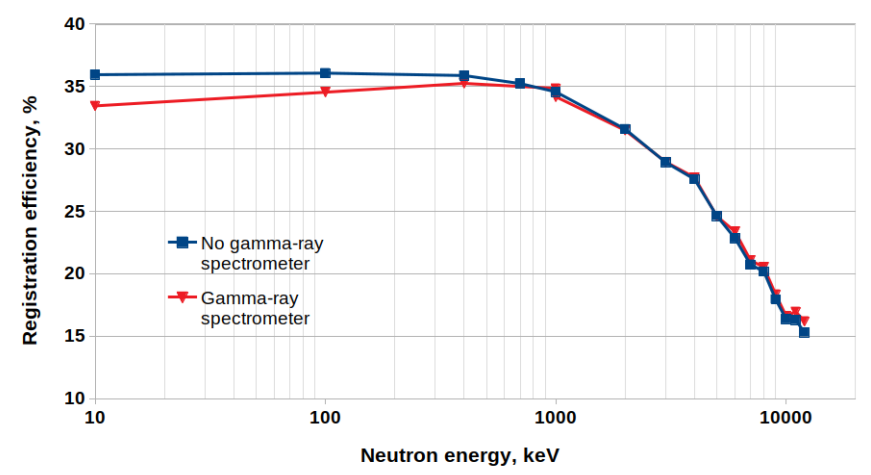

Fig. 7. Expected efficiency for 40 neutron counters based on Monte Carlo simulations corrected by measurements with ${ }^{252} \mathrm{Cf}$.

\section{CONCLUSIONS}

The MULTI spectrometer is designed for measurement of $\beta$ $\gamma$-neutron coincidences from the decay of exotic nuclei implanted into and active target. It consists of in-beam multidetector telescope for identification of secondary beam particles by the dE-TOF method, a gamma-ray spectrometer, and a $4 \pi$ neutron counter assembly. Radioactive nuclei are implanted into an active target, which provide particle identification and possibly information about $\beta$-particle energy. The active target will be surrounded by a $4 \pi$ scintillator phoswich detector for $\beta$ particles, capable of identification of high and low energy $\beta$ particles from the decay of implanted nuclei.

The gamma-ray spectrometer is assembled from nine $\mathrm{CeBr}_{3}+\mathrm{NaI}(\mathrm{Tl})$ phoswich scintillation detectors into a $3 \times 3$ cluster. The fundamental characteristics of the assembly were measured with a ${ }^{60} \mathrm{Co}$ spectroscopic source by a tagged gammarays method. It showed $2,5 \%$ detection efficiency for 1173,2 $\mathrm{keV}$ at a distance of $10 \mathrm{~cm}$ and are in relatively good agreement with simulations. Pulse-shape analyzing electronics can enhance performance of the spectrometer, when the $\mathrm{NaI}(\mathrm{Tl})$ crystal can be used as a Compton suppression detector. Measurements showed a $58 \%$ effect at a $40 \mathrm{~cm}$ distance from source, when mean height of the Compton part of the spectrum was lowered by factor 2,4 . The effect is even more significant at a $10 \mathrm{~cm}$ distance, achieving suppression of $63 \%$. This is important for gamma-ray spectroscopy measurements on background induced by high-energy gamma rays.

A $4 \pi$ phoswich scintillation detector for measurement of $\beta$ particles is currently being developed with help of Geant4 simulations. It will be able to distinguish between high and low energy beta particles, using pulse-shape analyzing electronics.

Multi-neutron emission following beta decay of exotic nuclei with large $\mathrm{Q}_{\beta}$ values will be measured with a neutron counter assembly located around the gamma-ray spectrometer. It consists of 40 proportional counters filled with ${ }^{3} \mathrm{He}$ gas. It covers a large solid angle, close to a $4 \pi$ geometry. The geometry was optimized with Geant4 simulations with respect to maximum detection efficiency. Results compared to measurements with a ${ }^{252} \mathrm{Cf}$ neutron source showed systematic error, resulting in a measurement-to-simulation factor of 0,67. Further improvements must take place before measurements on the ACCULINNA-2 fragment separator beam. According to Monte Carlo simulations corrected with experimental data, expected detection efficiency achieves value of $35 \%$ for a single neutron with energy up to $1 \mathrm{MeV}$.

The MULTI spectrometer will take place in measurements on ACCULINNA and ACCULINNA-2 fragment separators in the Flerov Laboratory of Nuclear Reactions of Joint Institute for Nuclear Research in Dubna, Russia. Moreover, the MULTI spectrometer is constructed to be portable. It can be optimized with validated Geant 4 simulations and deployed on beams at different laboratories.

\section{ACKNOWLEDGMENT}

This work was supported by Program of cooperation JINR Czech Republic (3+3) "Development of the MULTI-purpose spectrometer" $(2020,2021)$ and Grant of the Plenipotentiary of the Government of the Czech Republic in JINR "Total cross section study in reaction with exotic nuclei by MULTI setup".

\section{REFERENCES}

[1] Yu. G. Sobolev et al., "A setup for measuring total cross sections of nuclear reactions", Instruments and Experimental Techniques 55 (2012) pp. 618.

[2] A. S. Fomichev et al., "Studies of light exotic nuclei in the vicinity of neutron and proton drip lines at FLNR JINR", Phys.-Usp. 59 (2016) pp. 321.

[3] Yu. G. Sobolev et al., "Studying the excitation function of the full cross section of a reaction using a modified transmission technique: Initial results", Bulletin of the Russian Academy of Sciences: Physics 76 (2012) pp. 952.

[4] I. Sivacek et al., "Response function investigation of $4 \pi \gamma$-spectrometer "MULTI" by Geant4 Monte Carlo code", Proceedings of the International symposium on Exotic Nuclei: EXON 2016, Kazan, Russia, World Scientific, ISBN 9789813226531, pp. 356

[5] Yu. G. Sobolev et al., "Active collimators in experiments with exotic nuclear beams", Instruments and Experimental Techniques 54 (2011) pp. 449.

[6] Yu. G. Sobolev et al., "Measuring the Total Cross Sections for Reactions in Collisions of ${ }^{6,8} \mathrm{He}+{ }^{28} \mathrm{Si}$ and ${ }^{9} \mathrm{Li}+{ }^{28} \mathrm{Si}$ ", Bulletin of the Russian Academy of Sciences. Physics 83 (2019) pp. 402.

[7] Yu. G. Sobolev et al., "Total Reaction Cross Sections for ${ }^{6,8} \mathrm{He}$ and ${ }^{9} \mathrm{Li}$ Nuclei on ${ }^{28} \mathrm{Si},{ }^{59} \mathrm{Co}$, and ${ }^{181} \mathrm{Ta}$ Targets", Bulletin of the Russian Academy of Sciences. Physics 84 (2020) pp. 948. 
[8] Yu. E. Penionzhkevich et al., "Energy dependence of the total cross section for the ${ }^{11} \mathrm{Li}+{ }^{28} \mathrm{Si}$ Reaction", Phys. Rev. C 99 (2019) 014609.

[9] I. Sivacek et al., "MULTI-2, a $4 \pi$ spectrometer for total reaction cross section measurements", Nucl. Instr. Meth. A 976 (2020) 164255.

[10] I. Sivacek et al., "Upgrade possibilities of the spectrometer MULTI", Proceedings of the International Symposium on Exotic Nuclei EXON 2018, World Scientific, ISBN 9811209448, pp. 444.

[11] G. Kamynski et al., "Status of the new fragment separator ACCULINNA-2 and first experiments", Nucl. Instr. Meth. B 463 (2020) pp. 507.

[12] D. Testov et al., "The ${ }^{3} \mathrm{He}$ long-counter TETRA at the ALTO ISOL facility", Nucl. Instr. Meth. A 815 (2016) pp. 96.

[13] D. Testov et al., "Pygmy Gamow-Teller resonance in the $\mathrm{N}=50$ region: New evidence from staggering of $\beta$-delayed neutron-emission probabilities", Phys. Rev. C. 95 (2017) 054320.

[14] A. P. Severyukhin et al., "Pygmy dipole resonance of ${ }^{134} \mathrm{Sn}$ ", Proceedings of the International Symposium on Exotic Nuclei EXON 2018, World Scientific, ISBN 9811209448, pp. 341.

[15] S. Agostinelli, et al., "Geant4 - a simulation toolkit", Nucl. Instrum. Methods A 506 (2003) pp. 250.

[16] J. Allison, et al., Geant4 developments and applications, IEEE Trans. Nucl. Sci. 53 (2006) pp. 270-278

[17] J. Allison, et al., Recent developments in Geant4, Nucl. Instrum. Methods A 835 (2016) pp. 186-225.

[18] F. Camera, A. Maj, PARIS White Book, Published by the Henryk Niewodniczański Institute of Nuclear Physics, Polish Academy of Sciences, Kraków 2021, ISBN 978-83-63542-22-1

[19] M. Dakowski et al., "Reconstruction of particle multiplicity distributions using the method of statistical regularization", Nucl. Instr. Meth. 113 (1973) pp. 195. 